\title{
Two-choice and three-choice probability learning in fish'
}

ROBERT MARRONE AND SELBY EVANS

TEXAS CHRISTIAN UNIVERSITY

Employing an apparatus which required a simple swimming response and a spatial discrimination, 10 African mouthbreeders were tested in two-target $80: 20$ and 30:70 problems, a three-target 20:60:20 problem with guidance procedure, and a 20:60:20 problem with a non-correction procedure. The sequential probability ratio test was employed in the analyses of choice data. Results showed statistically significant matching in both two-target problems, and in the three-target problems both with and without guidance procedure.

In two-choice probability learning problems employing guidance procedure, matching behavior has been demonstrated for African Mouthbreeders (Bitterman, Wodinsky, \& Candland, 1958; Behrend \& Bitterman, 1961). Mouthbreeders were trained to strike at two Plexiglas targets which were illuminated with colored lights or patterns projected upon them. In a series of experiments with a confounded visual-spatial discrimination under guidance procedure, matching appeared in $80: 20,70: 30,60: 40$, and 50:50 problems. When guidance was replaced by a non-correctional procedure, matching gave way to maximizing. Choice behavior, under conditions of matching, appeared to be free of sequential dependency.

Observations by this $E$ have shown: (1) that the response of butting or striking an object has a very low frequency of occurrence for mouthbreeders, and (2) that their activity level is greatly affected by changes in illumination. It is possible that these factors increased variability in these studies and obscured choicebehavior characteristics of interest. The response required of Ss in the present study was a simple swimming response; $S$ swam through the alley of its choice and was subsequently rewarded or non-rewarded without change in illumination.

Regrettably, conclusions in the above studies were reached without benefit of statistical tests. The present study introduces to this area of research the sequential probability ratio test (Hoel, 1954). The chief merits of this analytical procedure in the context of the present study are that: (1) it allows $E$ to set both $\propto$ and $\beta$ level; (2) a relatively short testing period is required before a decision as to the rejection or acceptance of the hypothesis in question can be made; (3) either the matching hypothesis or the maximizing hypothesis is accepted before the data-taking ends; and (4) a separate decision is made for each $\mathrm{S}$. The sequential method of determining which of a set of two exact hypotheses is to be accepted consists of taking an observation and then making one of three decisions: (1) accept $\mathrm{H}_{1}$; (2) accept $\mathrm{H}_{2}$ (the alternative hypothesis); (3) further data are necessary to make a decision with sufficiently small risk of error. When the third decision is made, an additional observation is taken and the same three decisions are reconsidered. In the present application, instead of the usual one set of alternative hypotheses, two sets were tested. One such set of hypotheses concerned the matching value versus chance level responding, whereas the second set of hypotheses concermed the matching value versus the maximizing value.

The present experiment investigated the performance of African mouthbreeders in two-choice problems with guidance and in a three-choice problem both with and, subsequently, without guidance procedure.

\section{Method}

The Ss, 10 African mouthbreeders (Tilapia macrocephala), were approximately 2 in. in length and experimentally naive.

The apparatus, constructed of 1/4-in. Plexiglas, contained three partitioned alleys and a start area. Guillotine doors at the entrance of each alley allowed $E$ to block S's entrance to an incorrectly chosen alley on a guided trial. The apparatus was submerged in a glass aquarium which also housed Ss throughout the course of the experiment.

Following pre-training, Ss were trained in a 50:50 problem and tested subsequently in an 80:20 problem with guidance (G), a $30: 70$ problem (G), a three-alley 20:60:20 problem $(G)$, and a 20:60:20 problem without guidance (NG). The guidance procedure entailed the following: if on any choice trial the unreinforced alley was chosen, it was blocked on the subsequent guided trial and $S$ was permitted to earn a reward for response to the positive alley. Ss received 20 massed trials per

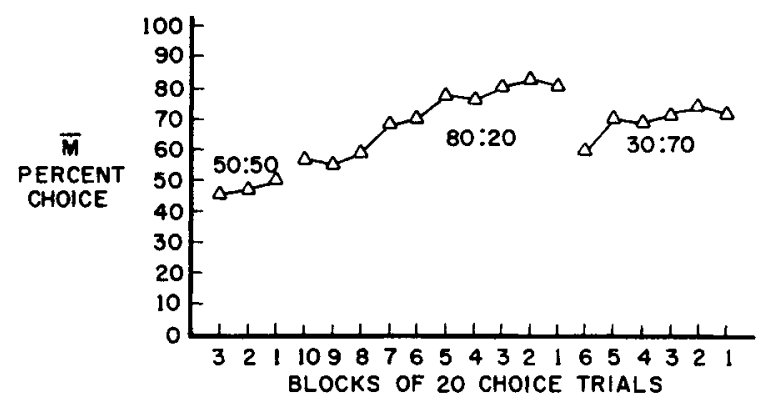

Fig. 1. $\Pi$ percentage of choices of the MFR alternative over the final blocks of 20 trials in the 50:50, 80:20, and 30:70 problems for all Ss. 
day, earning one pellet of food for either a correct choice on the choice trial or for a guided response to the positive alley on a guided trial.

\section{Results and Discussion}

All Ss met the criteria of matching in the 50:50, 80:20 and 30:70 problems. Plotted in Fig. 1 is the grand mean $(\bar{M})$ percentage of responses to the most frequently rewarded (MFR) alternative in these problems for all Ss. Because individual Ss met decision criteria under the sequential sampling procedure after a different number of days on each problem, only the final days of training are presented for the 80:20 and 30:70 problems. For each $\mathrm{S}$, the block numbers were determined by counting backward from the block at which the problem was terminated. The number of blocks for which data are reported is the maximum number of blocks for which data are available for all Ss.

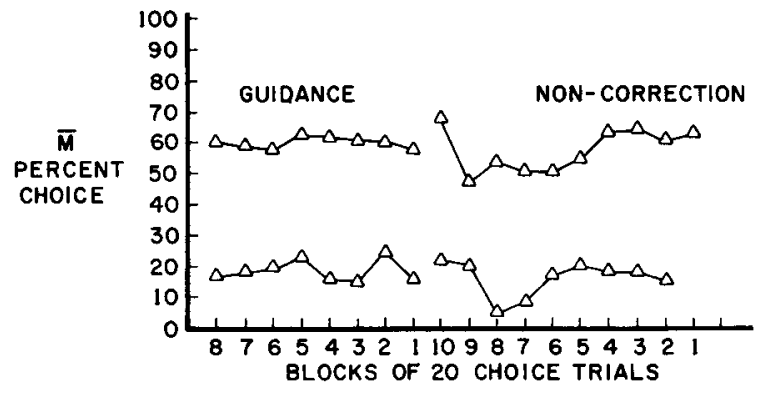

Fig. 2. $\bar{M}$ percentage of choices of the MFR and one other alternative for the final blocks of choice trials in the 20:60:20 problem, both with and without guidance procedure.

On completion of the 30:70 problem, Ss were tested in the three-choice 20:60:20 problem both with and, subsequently, without guidance. Five randomly chosen Ss were tested under the non-guidance condition. Under the sequential test procedure, decisions as to matching or non-matching were based on responses to the middle alley. All Ss met criteria of matching in the 20:60:20 problems, both with and without guidance procedure.

In Fig. 2 are plotted the $\bar{M}$ percentage of responses to the MFR alley and to one alternative alley for the 20:60:20 problem, both with and without guidance. The same procedure for determining the maximum number of blocks in which all Ss participated was used in this problem as was employed in the 80:20 and 30:70 problems. It appears from Fig. 2 that matching in the three alley situation was with respect to all three alternatives and not merely the MFR alley.

Although no formal analytic procedure for ascertain- ing the existence or non-existence of sequential dependencies in the choice behavior of Ss was employed in the present experiment, examination of cumulative choice curves did not suggest such dependencies.

The results of the present experiment thus support three findings of the Bitterman $(1958,1961)$ experiments: (1) mouthbreeders demonstrate matching behavior in spatial problems under guidance procedure; (2) matching is an individual, not merely a group phenomenon; and (3) inspection does not indicate the presence of sequential dependencies in choice behavior under conditions of matching.

Other findings of interest in the data from the present experiment are: (1) mouthbreeders match reward ratios in a three-choice problem and this matching is to all three alternatives; (2) choice behavior in the threechoice problems also reveals a virtual absence of sequential dependencies upon observation; and (3) these characteristics of matching in the three-choice problem are evidenced both under a guidance procedure and under a non-correctional procedure.

In an effort to determine whether the experimental procedures in the present study did, as expected, reduce variability relative to that produced by the procedures of Behrend \& Bitterman (1961), data presented graphically in that study was used to estimate the variance of daily group means about the grand mean for the final 100 trials of the 70:30 and of 80:20 problems. From the present data, eight Ss were chosen at random to equalize $\mathrm{N}$, and comparable SD estimates were obtained (since these means should reflect primarily random variability). In both problems the variance obtained in the present study was about $25 \%$ of that found in the Behrend and Bitterman study.

The results suggest that (1) the present procedures may substantially reduce variability in performance; (2) further study is needed of the conditions producing matching behavior under the non-correction procedure; and (3) the sequential probability ratio test appears useful in this kind of study.

\section{References}

Behrend, Erika R., \& Bitterman, M. E. Probability-matching in the fish. Amer. J. of Psychol., 1961, 74, 542-551.

Bitterman, M. E., Wodinsky, J, \& Candland, D. K. Some comparative psychology. Amer. J. of Psychol., 1958, 71, 94-110.

Hoel, P. G. Introduction to mathematical statistics. New York: Wiley, 1954.

\section{Note}

1. This research was submitted in partial fulfillment of the requirements for a Master of Arts degree at Texas Christian University, 1965 . 